\title{
Sigmoidectomía Laparoscópica Reporte de los siete primeros casos en el Perú
}

\author{
ALEJANDRO BAZAN, LUTS POGGI, LUTS SALDARRIAGA, JAVIER SANDOVAL \\ Deparamento de Cirugía Flumana Facultad de Medicina, UNMSM. Servicio de Cirugía General N 3, HNGAI-IPSS
}

\begin{abstract}
RESLIIEN:
Se presenta los casos de siete pacientes sometidos a sigmoidectomía laparoscópica en el Servicio de Cirugía $N^{\circ} 3$ del Departamento de Cirugía General del Hospital Nacional «Guillermo Alnenara Irigoyen», durante Ios años 19931995. De los siete casos, cinco eran portadores de patología benigna (cuatro con dolicomegasigma y uno con adenoma velloso benigno) y dos presentaban patología tumoral maligna resecable. Se describe los procedimientos quirúrgicos practicados y se relata el post-operatorio inmediato, que en general fue safisfactorio.
\end{abstract}

Palabras claves: Colon sigmoide, cirugía laparoscópica, cinugia de colon y recto.

\section{LAPAROSCOPIC SIGMOIDECTOMY: REPORT OF THE 7 FIRST CASES PERFORMED IN PERU SUIMAARY}

VVe present seven cases of laparoscopic sigmoidectomy performed at the Surgical Service $\mathrm{N}^{\circ} 3$, Department of General Surgery, Guillermo Almenara Irigoyen National Hospital betw'een 1993 and 1995. Five had benign pathology (four had dolicomegasigma and one benign villous adenoma) and two presented resectable malignant tumor pathology. We describe the surgical procedures and the usually satisfactory immediate post-operative period.

Rey words: Sigmoid colon, surgical Laparoscopy, colon and rectal surgery.

\section{INTRODUCCION}

El advenimiento de la cirugía laparoscópica con sus múltiples ventajas, puestas en evidencia primeramente en la colecistectomía y luego en otros procedimientos quirúrgicos abdominales, como la hemioplastía y cura quirúrgica del reflujo gastroesofágico, incentivaron a las escuelas quirirgicas americanas de Miami y Texas a introducir esta técnica a la cingǵa colónica a partir de 1992, efectuando resecciones de colon para patología benigna y posteriormente maligna.(123.3.72) En el Servicio de Cirugía General № 3 de nuestro hospital, se inicia la práctica de este procedimiento a partir de 1993, después de alcanzar amplia expericncia en las colecistectomías laparoscópicas y de pertrecharnos de un adecuado arsenal de instrumental quirúrgico laparoscópico necesario, que en el curso de dos años ha ido modernizándose progresivamente. Aún cuanđo Geis $\left(^{4}\right)$ sostiene que la hemicolectomía derecha es menos complcja que la sigmoidectomía con la técnica de laparoscopía asistida, nosotros hemos iniciado las resecciones sigmoideas laparoscópicas, esta escala de graduación de complejidad de Geis esta referida a la práctica o no de

Correppondenciu.

Dr. Alejundro Bazün Gınzáles.

Fucultad de Medicina U. N.M.S.M.

A. Grau 755. Lima I - Perí movilización, desvascularjzación y anastomosis intracorporales.

En el prescnte artículo se expone la historia clínica suscinta de los siete casos de sigmoidectomía laparoscópica realizados en el Servicio, enfatizando en la técnica quirúrgica y la evolución postoperacoria imınediata, que aún cuando constituycn una serie corta, resulta muy significativa para el acúmulo de experiencia y permite avisorar un porvenir seguro para el manejo de esta técnica aplicada a la cirugía colónica

\section{PROCEDIMIENTO}

Preparación del paciente: La evaluación pre-operatoria se practica de acuerdo al protocolo para esta clase de pacientes. Se les hospitaliza dos días antes del acto operatorio. La limpieza del colon se inicia 48 horas antes con dieta líquida y el día anterior, a partir de las 14 brs, se suministra vía oral solución de pofietilenglicol (PEG) y, en ausencia de este producto, manitol al $20 \%$, hasta obtener deposiciones completamente líquidas y acuosas. Se repone líquidos y electrolitros endovenosos según el criterio clínico. Usamos metronidazol $500 \mathrm{mg}$. TV al momento de la inducción anestésica.

Procedimiento operatorio: Bajo anestesia general -intubado, se le coloca al paciente sonda nasogástrica y de Foley en vejiga, se le acomoda en posición ginecológica con pierneras a media altura para permitir el fácil abordaje de la región anoperineal y a la vez un cómodo acceso al abdomen. 
Previa asepsia y antisepsia se coloca aguja de Veress en cicatriz umbilical y se procede a realizar el ncumoperitoneo usanclo un insullador electrónico hasta alcanzar un valor de presión intraabciominal de $15 \mathrm{~mm}$ de $\mathrm{Hg}$, luego de lo cual se introduce el trocar de $11 \mathrm{~mm}$ que da paso al laparoscopio de $0^{\circ}$ para explorar todo el abdomen. Después se introduce tres trócares de $12 \mathrm{~mm}$, dos en hemiabdomen izquierdo (uno subcostal en línea medio clavicular y ofro en línea axilar anterior, ligcramente supraumbilical) y uno suprapúbico sulïcientemente lateralizado a la derecha. Fig. 1.

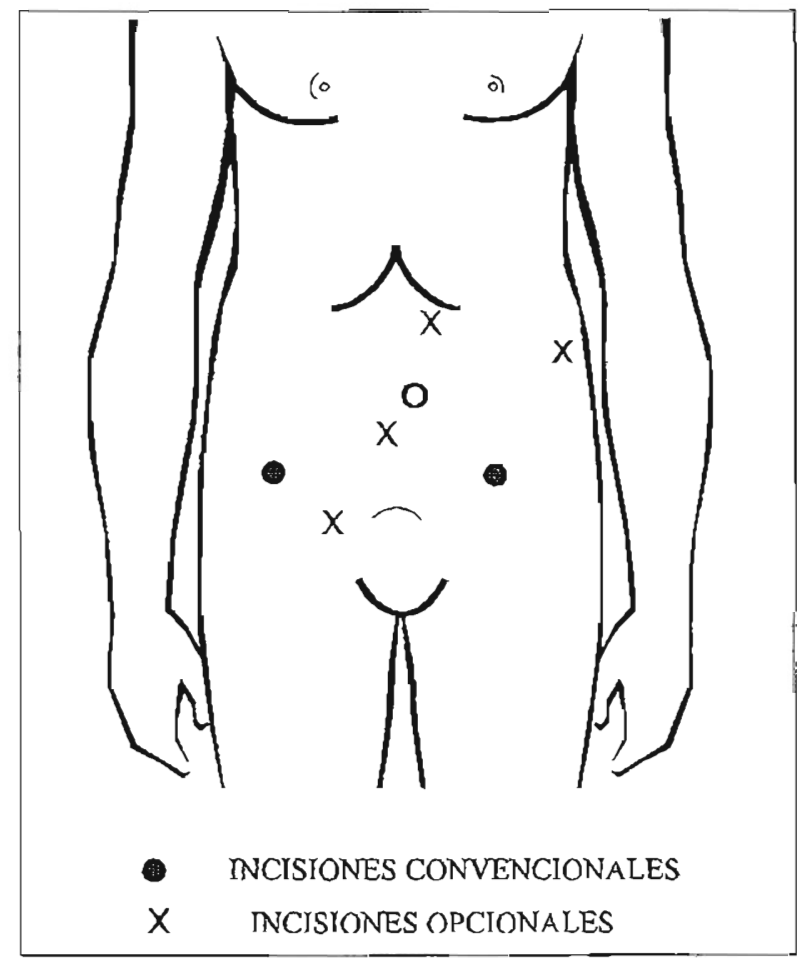

Fig. N"1.

Se coloca al paciente en posición de Trendelenburg máxima a fin de desplazar facilmente las asas delgadas hacia el diafragma, «desocupar» la región pélvica y visualizar convenientemente el rectosigmoides. Se pasa el endoscopio a la incisión superior izquierda: el cirujano trabaja por las incisiones umbilical y suprapúbica y el asistente colabora a través de la incisión interior izquierda. Se expone el sigmoides mediante tracción con endograsper o endobabcock y se procede a incidir y decolar el parietocólico izquierdo con endoshears (rijeras) usando corle y coagulación. En este paso debe ubicarse el ureter y los vasos ilíacos. Se diseca el mesosigmoides; este es un tiempo a veces muy difícil en el dolicomegasigma por la mesenteritis consecutiva a episodios previos de semivolvulación $\left({ }^{12}\right)$. Se diseca el meso y se ubica los vasos sigmoideos los cuales se clipan individualmente usando clips "large" de titanio con clipadora mecánica o automática. También pueden ser ligados y cortados en bloque con endo-GIA. 30, lo que hace más fácil y rápido el procedimiento, aunque muy oneroso.
También pucue usarse cndoloops para reforzal cl clipado de la arteria mesencérica inlerior, esro abarala más aún el procedimienlo. La sección del sigmoides se hace con endo GIA N 60 (VERDE) que se introduce por la incisión suprapuíbica, colocando trócar 15' (lïg. 2). El segrmento colónico a resecar se exurae por la incisión şuprapúbica, siendo neccsario ampliarla a $3 \mathrm{~cm}$. (fig. 3). Se secciona el scgmento colónico en lá longitud deseada y se practica sutura circular (en coronis) contínua con nylon $000 \mathrm{cn}$ cl cxtremo proximal seccionado, palra lija! la hemiestcra clistal del Stapler (cartucho CEEA No 31) que se usará para la anastomosis colorcctal.

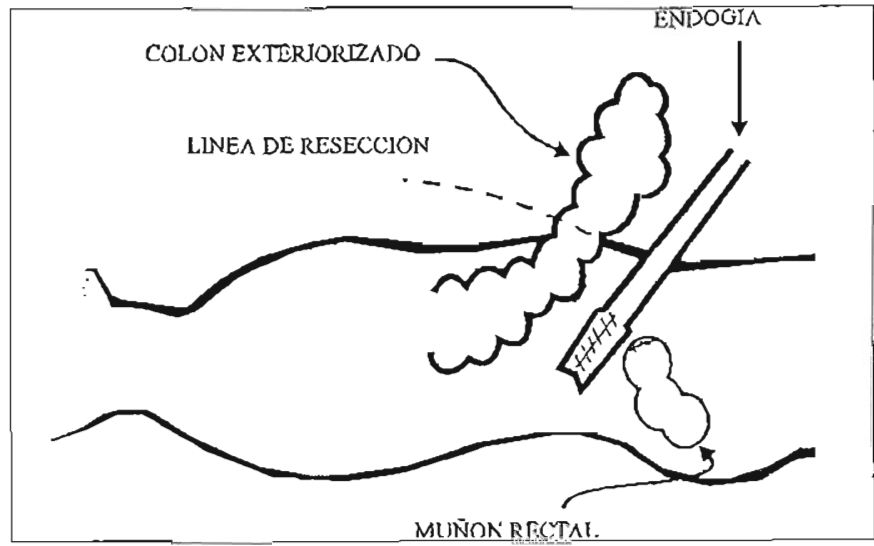

Fig. N. 2.

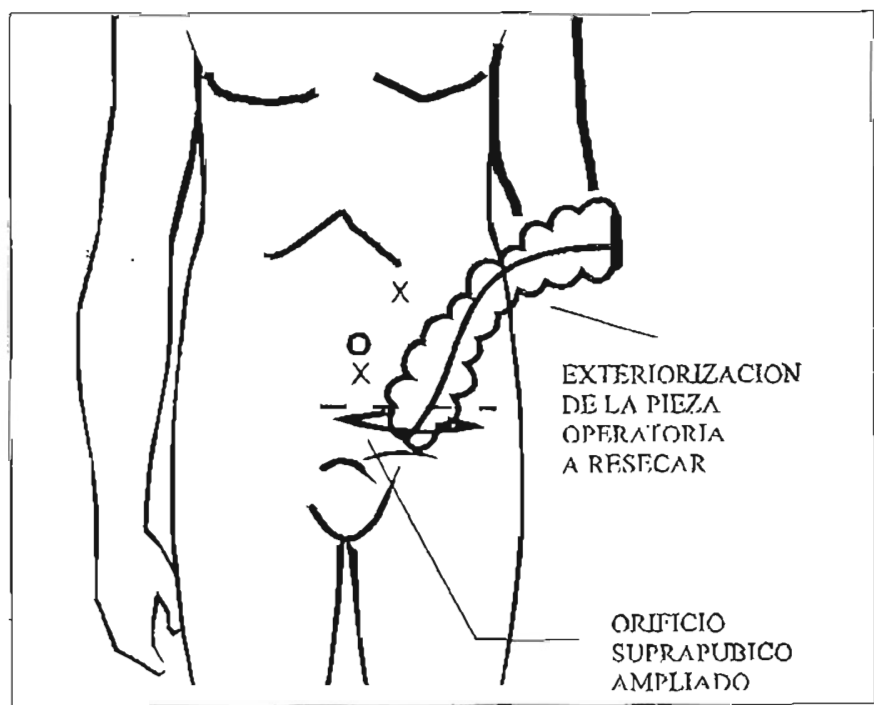

Fig. N" 3.

Se devuelve a la cavidad el muñón colónico proximal y se cierra la incisión por planos usando nylon I en surger para la aponeurosis. Se reinstala el neumoperitoneo y se introduce por el ano un Stapler (curvo) CEEA N 31 (verde) que contiene la hemiestera proximal hasta alcanzar el vértice del inuñón distal. Se realiza la anastomosis colónica rectal termino-lateral mediante disparo del CEEA (lig. 4). 


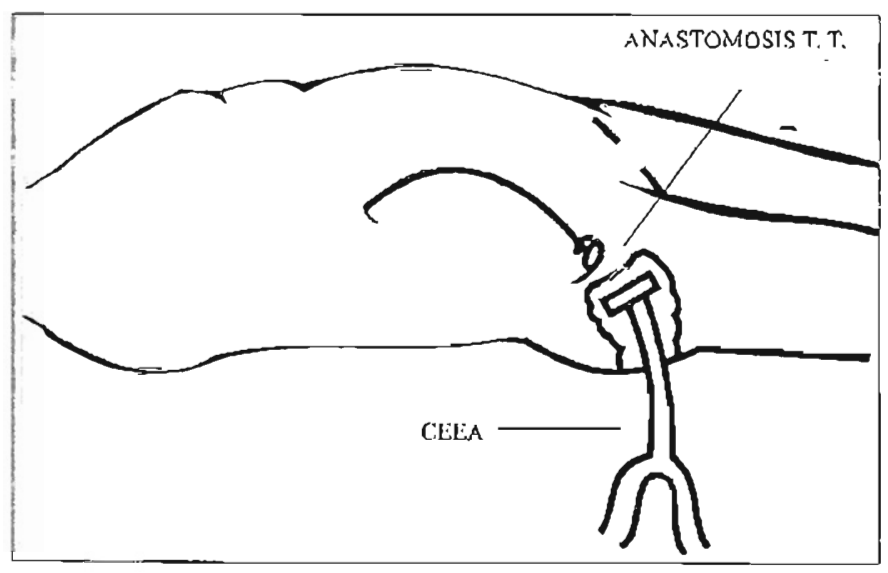

Fig $N$

Se prueba la sutura insuflando aire por vía rectal, luego se lava y Gena (si se estima conveniente) el lecho pélvico con sonda de nélaton $\aleph^{*} 16$ que se exterioriza por la incisión suprapúbica. En ningún caso practicamos la sigrmoidectomia laparoscópica total con extracción transanal de la pieza operatoria extirpada $\left({ }^{2}\right)$, técnica cuyas ventajas ha demostrado Lirici (") en cirugía laparoscópica experimental.

\section{PRESENTACION DE LOS CASOS}

A continuacion presentamos la historia clínica de los siete pacientes sigmoidectomizados:

Caso 1:

Paciente: R. R.

Edad: 26 años

Sexo: Femenino

Paciente con I.R.C. en diálisis peritoneal ambulatoria. Presenta dolor y distensión abdominal severa. $\mathrm{Rx}$ simple de abdomen: signos compatibles con vólvulo de sigmoides.

Preparación: Manital 20\%. Operación: (nov. 1993) resección laparoscópica asistida, anastomosis T.T. (Extra abdominal). Continuó en diálisis peritoneal. Tiempo operatorio 5 hrs. 30' Evolución: Favorable

\section{Caso 2:}

Paciente: E. C. A.

Edad: 72 Años

Sexo: Femenino

Tiempo de enfermedad: 1 An̆o

Diarreas frecuentes, pérdida de moco y sangre en las heces.

Colonoscopía: adenoma velloso a $25 \mathrm{~cm}$. de la margen anal.

Preparación: Manitol al 20\%. Operación sigmoidectomía laparoscópica. Tiempo operatorio: 6 hrs. 05'. Alta: 4to. Día.

Caso 3:

Paciente: C. A. C.

Edad: 67 Años

Sexo: Masculino.

Paciente con episodios de obstrucción intestinal, resueltos con manejo médico pasando sonda rectal. Dx dolicomegasigma Preparación: Polietilenglicol.(P.E.G)
Operación: Sigmoidectomía laparoscópica.

Tiémpo operatorio: 5 hrs. 10'.

Alca: 5to. día.

Caso 4:

Paciente: M. M. R.

Edad: 81 años

Sexo: Femenino.

Episodios de deposiciones semiliquidas, sanguinolentas con moco, pujo y tenesmo rectal.

Proctoscopía: Tumor estenosante a $35 \mathrm{~cm}$ de margen anal. Biopsia: adenocarcinoma tubular bien diferenciado de sigmoides.

Preparación: Polietilenglicol.

Operación: (18-11-94) Sigmoidecomía + colostomía a lo Harcmann laparoscópica. Tiempo operatorio: 4 hrs. 50'

Alta: $10 \mathrm{mo}$. día.

\section{Caso 5:}

Paciente: H. D. D.

Edad: 30 años

Sexo: Masculino

Ingresa por emergencia, se diagnostica vólvulo de sigmoides, se desvolvula con sonda rectal. Se le programa para cirugía electiva

Preparación: Polietilenglicol.

Operación: (06-04-95). Sigmoidectonía laparoscópica.

Tiempo operatorio: 4 hrs. 35 '.

Alta: 8vo. día.

Caso 6:

Paciente: J. S. D.

Edad: 75 años

Sexo: Masculino

Antecedentes de Ca de próstata (un año) sometido a orquiectomía bilateral y terapia hormonal. Inmediatamente después, episodios de dolor abdominal difúso tipo cólico con distensión, constipación y dificultad para eliminar gases, que cedía con automedicación. Ingresa por emergencia. Rx de colon con enema barilada. Dolicosigma, además uná neoplasia bien localizada en sigmoides sin ganglios metastásicos visibles. Operación: (20-08-95) sigmoidectomía laparascópica. Tiempo operacorio: 4 jrs. 25'. Sepsis Peritoneal: Laparotomía a las 72 hrs. post-operatorio.

Caso 7:

Paciente: R. L. C

Edad: 72 años.

Sexo: Masculino

Antecedentes: Diabetes mellitus, laparotomía por peritonitis, colecisleclomía. Presentó dos episodios de obstrucción intestinal baja. Rx de colon con enema a doble contraste: dolicomega colon y ent. diverticular. La obstrucción aguda se resuelve mediante proctosigmoidoscopía.

Cirugía Elecliva: Sigmoidectomía ampliada laparoscópica.

Tiempo operatorio: 5 hrs. 20 '.

Complicaciones: Infección de herida operatoría en flanco izquierdo (zona cle exteriorización del sigmoides) 


\begin{tabular}{|c|c|c|c|c|c|c|c|c|}
\hline$N^{0}$ & Paciente & Edad & Sexo & Diagnóstico & Preparación & Cirugía & $\begin{array}{c}\text { Ticmpo } \\
\text { Operatorio }\end{array}$ & Complicaciones \\
\hline 1 & R.R & 26 & $F$ & $\begin{array}{l}\text { Vólvulo } \\
\text { sigmoides }\end{array}$ & Manitol 20\% & $\begin{array}{l}\text { Sigmoidectomía } \\
\text { laparoscópica } \\
\text { asistida } \\
\text { Anastomosis } \\
\text { extra abdoninal }\end{array}$ & 5 h. $30^{\prime \prime}$ & \\
\hline 2 & E.C.A. & 72 & $\mathrm{~F}$ & $\begin{array}{l}\text { Adenoma } \\
\text { velloso }\end{array}$ & Manitol 20\% & $\begin{array}{l}\text { Sigmoidectomía } \\
\text { laparoscópica }\end{array}$ & 6 เ. $05^{\prime \prime}$ & \\
\hline 3 & C.A.C. & 67 & M & $\begin{array}{l}\text { Vólvulo } \\
\text { sigmoides }\end{array}$ & P. E. G. & $\begin{array}{l}\text { Sigmoideciomía } \\
\text { laparoscópica }\end{array}$ & 5 h. $10^{\prime \prime}$ & \\
\hline 4 & M.M.R. & 81 & M & $\begin{array}{l}\text { Adeno } \\
\text { carcinoma }\end{array}$ & P. E. G. & $\begin{array}{l}\text { Sigmoideciomía } \\
\text { + colostomía a lo } \\
\text { Hartmann } \\
\text { laparoscópica }\end{array}$ & 4 h. $10^{\prime \prime}$ & \\
\hline 5 & H.C.D. & 30 & M & $\begin{array}{l}\text { Vólvulo } \\
\text { sigmoides }\end{array}$ & P. E. G. & $\begin{array}{l}\text { Sigmoidectomía } \\
\text { laparoscópica }\end{array}$ & 4 ।. $35^{\prime \prime}$ & $\begin{array}{l}\text { Infección de herida } \\
\text { suprapúbica. }\end{array}$ \\
\hline 6 & J.S.D. & 75 & $M$ & $\begin{array}{l}\text { Adeno } \\
\text { carcinoma }\end{array}$ & P. E. G. & $\begin{array}{l}\text { Sigmoidectomía } \\
\text { laparoscópica }\end{array}$ & 4 ให. $25^{\prime}$ & Sepsis peritoneal \\
\hline 7 & R.L.C & 72 & M & $\begin{array}{l}\text { Vólvulo de } \\
\text { sigmoides } \\
\text { ent. diverticular }\end{array}$ & P.E. G. & $\begin{array}{l}\text { Sigmoideclomía } \\
\text { laparoscópica } \\
\text { aplicada }\end{array}$ & 5 म $20^{\circ}$ & $\begin{array}{l}\text { Inleccion de herida } \\
\text { flanco izquierdo }\end{array}$ \\
\hline
\end{tabular}

\section{DISCUSION}

La cirugía colónica laparoscópica esta sometida a proceso altamente selectivo, Ramos ('1)), de una serie de 200 casos, sólo eljgió 62 . de ellos 24 fueron sigmoidectomías. Fine $\left({ }^{3}\right)$, en su serie de 30 colectonías, refiere que siete correspondieron a sigmoidectomía.

En nuestra serie, las edades que superan la séptima década corespondieron a portadores de patologías tumorales (dos adenocarcinomas y un tumor velloso), dos de los cuatro pacientes con vólvulo sigmoideo presentaron edades por debajo de la cuarta década. E! predominio sexual correspondió al masculino (cuatro pacientes).

El diagnóstico preoperatorio fue acertado en cinco casos. Un paciente con terapia hormonal por cáncer de próstata ingresó a sala con diagnóstico de vólvulo de sigmoides y el hallazgo operatorio resultó un adenocarcinoma estenosanie, lo que evidencia que su estudio diagnóstico fue deficitario. En la serie de Plasencia $\left({ }^{8}\right)$, de treinta pacientes, 18 eran portadores de cáncer y 12 de lesiones benignas

Destacamos la alta calidad de la limpieza del colon que brinda el polietilenglicol (PEG) que se usó en cuatro casos; no compromete el equilibrio hidroelectrolílico del paciente, no proúuce distensión de asas, ni riesgo de explosión con el uso de electrocauterio. Supe- ra ampliamente las virtudes del manitol al 20\% que se adininistró en dos pacientes.

El abordaje quirúrgico to practicamos mediante cuatro incisiones, una umbilical, una suprapúbica lateralizada a la derechil y dos incisiones izquierda. una cicbajo del reborde costal en la línea medio-clavicular y otra en la linea axilar anterior ligeramente supra umbilical. La incisión suprapúbica primero deja pasar un trócar $\mathrm{N}^{\circ}$ 12 y luego un trócar $\mathrm{N}^{\circ} 15$; debe ubicarse suficicntemente lateralizada para permitir maniobrar el endo-GIA \# 60 (verde) que realiza la sección distal del sigmoídes y cierra el muñón rectal. En dos casos se introdujo el procloscopio para ayudarnos a visualizar el recio y determinar el nivel de resección. En ningún caso usamos la técnica de los alambres de Kirschner complementadia con minilaparotomía izquicrda $\left({ }^{15}\right)$.

En cinco casos fue posible la sigmoidectomía laparoscópica asistida. En un paciente se practicó anastomosis colónica termino terminal manual extracorpórea ampliando la incisión suprapúbica a $5 \mathrm{~cm}$. Puede también recurrirse a la incisión opcional en fosa iliaca izquierda (Fig. 5 y 6). En otro paciente. por rasgadura del muñón rectal, se realizó colostomia a lo Hartman laparoscópica- 
mente. Un paciente presentó sepsis peritoneal que necesitó laparotomía a las 72 horas, encontrándose lesión de pared de colon descendente de $0,5 \mathrm{cms}$ de diámetro. Se refiere como morbilidad hasta $6,3 \%$ de complicaciones $\left({ }^{(1)}\right)$, entre las más graves se anota la lesión de la arteria iliaca $\left({ }^{7}\right)$, Schulze refiere un exitus letalis en su serie de 8 casos.

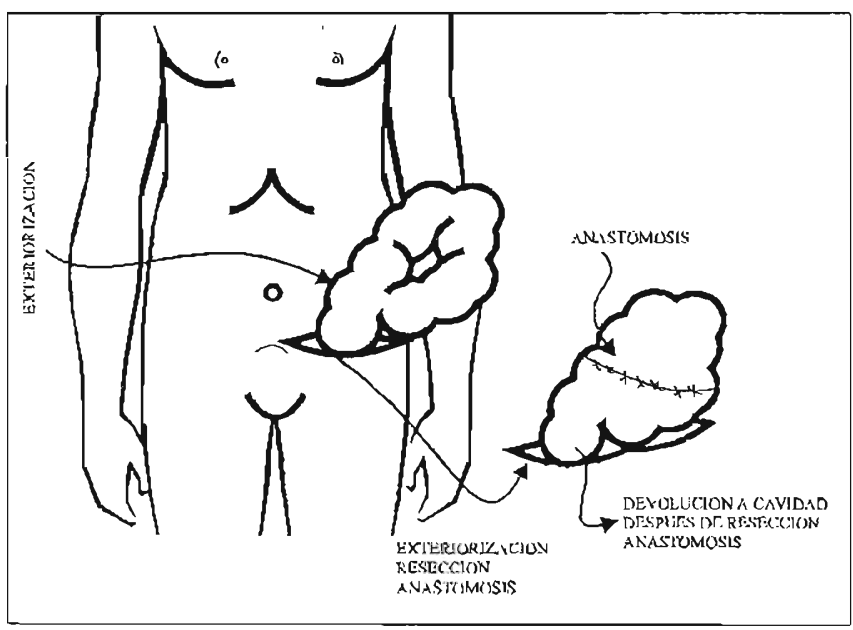

Fig. $N^{\prime \prime} 5$.

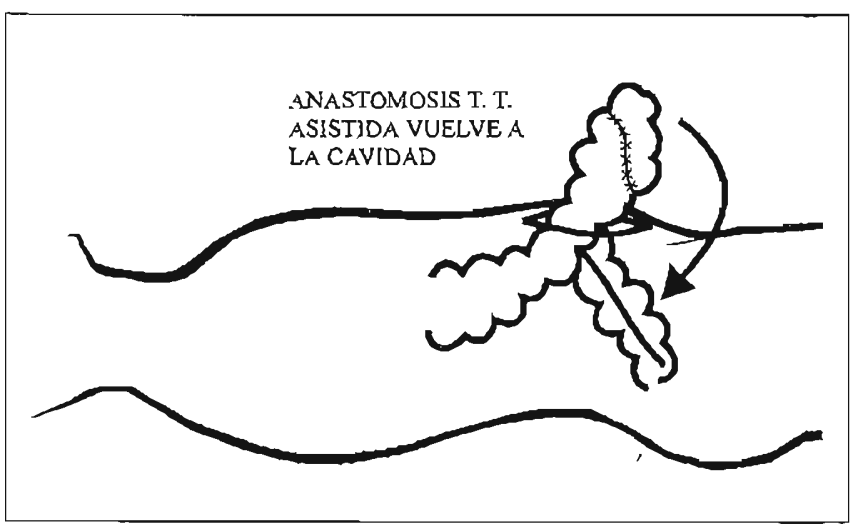

Fig. $N^{\prime \prime} 6$.

La conversión de cirugía laparoscópica a convencional en la patología del colon alcanza el $10 \%\left({ }^{3}\right)$ y, en algunas series, se refiere hasta $22 \%$ de conversión debido a tumores muy extensos o múlliples; y por adherencias rebeldes por cirugía previa (") se llega al $25 \%$ (").

Un dato valioso es la reducción progresiva del tiempo operatorio conforme se consigue mayor familiaridad con la técnica (de 6 hrs $10^{\prime}$ a 4 hrs $25^{\prime}$ ) y el comfort post-operalorio por las incisiones mínimas que se practica. El alta fue más precoz comparada con la técricá convencional, excepto en dos casos en que ocurrió infección de la herida supra-púbica en uno de sepsis peritoneal que obligó a la laparotomía en otro. La rebaja de los costos según un estudio multicéntrico se redujo de 13,050 a 11,010 dólares $\left(^{3}\right)$. El postoperatorio promedio alcanza 4 días $\left(^{8}\right)$ a $4.8\left(^{(7)}\right.$.

\section{CONCLUSIONES}

- Se presenta siete primeros casos de sigmoidectomía laparoscópica a nivel nacional. Gracias a la práctica adquirida en la colecistectomía laparoscópica y al manejo de la zona inguino-crural durante la práctica de hemioplastías, se ha incursionado con relativa pericia en la cirugía colónica laparoscópica

Los resultados son promisorios por el comfort que brinda al paciente, la consiguiente precocidad del alta y la evidencia de la progresiva reducción del tiempo operatorio.

\section{BIBLIOGRAFIA}

I) Ballantyne Leahy, Modlin. Laparoscopic Surgery. W. B. Saunders Coimpany. 1994

2) Darzi A, Supar P, Guitlon PJ, Monson J. Laparoscopic sigmoid colectomy, total laparoscopic apjproach. Discases of Colon and Rectum, 1994. 37, 268-271.

3) Finc AP, Lanasa $S$, Gannon MP, Cline AD, James R. Laparoscopic colon surgery, report of a series. American Surgery, 1995, 61, 5, 4l2-416.

4) Geis WP, Colctta AV, Verdia JC, Alasencin G, Diogho O, Jacibs M. Sequential psychomolor skills development in laparoscopic colon surgery. Archives of Surgery, 1994, 129, 206-212.

5) Kawamura J, Sart H, Sawada T, Mulot, Haga H. Laparoscopic assisted colectomy and lymphadenectomy without peritoneal insufflation for sigmoid colon cancer patients. Discases of Colon and Rectum. 1995; 38: 550-5.52.

6) Lirici MM, Huess G, Melyer A, Mcinreichs, Mcbrnann M, Bccker HD. Ncw Technique for sigmoid colectorny. British Joumal Suigery. 1993; 80: 1606-1609.

7) Morris E Franklin Jr. MD. Laparoscopic colonic procedures. World Jounal Surgery. 1993 Cop. 17 Pág. 51 - 56.

8) Plasencia G, Jacobs M, Verdeja JC, Viamonte M. Laparoscopic sssisted sigmoid colectomy und low anterior resection. Diseases of Colon and Recturn. 1994; 37: 829-833.

9) Puente I, Susa JL, Slecman D, Desai V, Tranakas N, Hartmann R. Laparoscopic assisred colorrectal surgery, Journal of Laparo-Endoscopic Surgery. Feb. 1994. Vol $4 N^{\circ}$ 1. Pág I - 7.

10) Ramos JM, Beart RW, Goes R, Ortega AE, Schlinkert RT, Role of laparoscopy in colecorrectal surgery a prospective evaluation of $200 \mathrm{ca}-$ ses. Diseases of Colon and Rectum. 1995; 38: 494-501.

(1) Scluulye S, stage JG. Laparoscopic colonic surgery. Ugesks-Laeger Tan 1994: 156: 640-643.

12) Sundin JA, Wass on D, Mc Millen MM, Ballantone G. Laparo-endoscopy Surgery, 1992; 2: 353-358. 